\title{
Bcl-2-like protein-10 increases aggressive features of melanoma cells
}

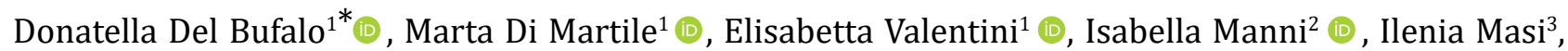

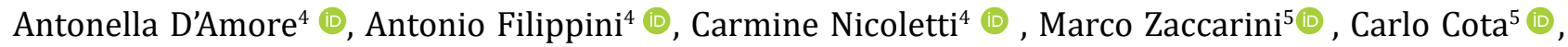
Maria Victoria Castro ${ }^{6,7}$, María Josefina Quezada ${ }^{6,7}$ (ㄷ) , Laura Rosanò ${ }^{1,3}$ (ㄷ) , Pablo Lopez-Bergami ${ }^{6,7}$ (1) , Simona D'Aguanno ${ }^{*^{*}}$ (1)

${ }^{1}$ Preclinical Models and New Therapeutic Agents Unit, IRCCS Regina Elena National Cancer Institute, 00144 Rome, Italy

${ }^{2}$ SAFU Unit, IRCCS Regina Elena National Cancer Institute, 00144 Rome, Italy

${ }^{3}$ Institute of Molecular Biology and Pathology, National Research Council, 00161 Rome, Italy

${ }^{4}$ Unit of Histology and Medical Embryology, Department of Anatomy, Histology, Forensic Medicine and Orthopaedics, Sapienza University, 00161 Rome, Italy

${ }^{5}$ Genetic Research, Dermatological Molecular Biology and Dermatopathology Unit, IRCCS San Gallicano Dermatological Institute, 00144 Rome, Italy

${ }^{6}$ Centro de Estudios Biomédicos, Básicos, Aplicados y Desarrollo, Universidad Maimónides, Buenos Aires C1405BCK, Argentina ${ }^{7}$ Consejo Nacional de Investigaciones Científicas y Técnicas, Buenos Aires C1405BCK, Argentina

*Correspondence: Donatella Del Bufalo, donatella.delbufalo@ifo.it; Simona D’Aguanno, simona.daguanno@ifo.it. Preclinical Models and New Therapeutic Agents Unit, IRCCS Regina Elena National Cancer Institute, Via Elio Chianesi 53, 00144 Rome, Italy

Academic Editor: Germain Gillet, Université de Lyon, France

Received: September 20, 2021 Accepted: December 20, 2021 Published: January 30, 2022

Cite this article: Del Bufalo D, Di Martile M, Valentini E, Manni I, Masi I, D’Amore A, etal. Bcl-2-like protein-10 increases aggressive features of melanoma cells. Explor Target Antitumor Ther. 2022;3:11-26. https://doi.org/10.37349/etat.2022.00068

\begin{abstract}
Aim: B-cell lymphoma-2 (Bcl-2)-like protein-10 (Bcl2L10) is the less studied member of Bcl-2 family proteins, with the controversial role in different cancer histotypes. Very recently, Bcl2L10 expression in melanoma tumor specimens and its role in melanoma response to therapy have been demonstrated. Here, the involvement of Bcl2L10 on the in vitro and in vivo properties associated with melanoma aggressive features has been investigated.
\end{abstract}

Methods: Endogenous Bcl2L10 protein expression was detected by western blotting analysis in a panel of patient-derived and commercially available human melanoma cells. In vitro assays to evaluate clonogenicity, cell proliferation, cell migration, cell invasion, and in vitro capillary-like structure formation [vasculogenic mimicry (VM)] have been performed by using human melanoma cells stably overexpressing Bcl2L10 or transiently transfected for loss/gain function of Bcl2L10, grown under two- or three-dimensional (3D) conditions Xenograft melanoma model was employed to evaluate in vivo tumor growth and angiogenesis.

Results: Results demonstrated that Bcl2L10 acts as an inducer of in vitro cell migration, invasion, and VM, while in vitro cell proliferation, in vivo tumor growth, as well as colony formation properties were not affected. Dissecting different signaling pathways, it was found that Bcl2L10 positively affects the phosphorylation of extracellular-signal-regulated kinase (ERK) and the expression of markers of cell invasion, such as urokinase plasminogen activator receptor (UPAR) and matrix metalloproteinases (MMPs).

(C) The Author(s) 2022. This is an Open Access article licensed under a Creative Commons Attribution 4.0 International License (https://creativecommons.org/licenses/by/4.0/), which permits unrestricted use, sharing, adaptation, distribution and reproduction in any medium or format, for any purpose, even commercially, as long as you give appropriate credit to the original author(s) and the source, provide a link to the Creative Commons license, and indicate if changes were made. 
Of note, Bcl2L10-dependent in vitro migration, invasion, and VM are linked to uPAR. Bcl2L10 also negatively regulates the intracellular calcium level. Finally, reduced invasion capability in 3D spheroid invasion assay of melanoma cells transiently overexpressing Bcl2L10 was observed after treatment with inhibitors of MMPs and UPAR.

Conclusions: Overall, data reported in this paper provide evidence supporting a positive role of Bcl2L10 in melanoma aggressive features.

\section{Keywords}

B-cell lymphoma-2-like protein-10, melanoma, invasion, migration, vasculogenic mimicry

\section{Introduction}

Cutaneous melanoma is one of the most aggressive forms of skin cancer, characterized by high mortality rate, metastases, and resistance to conventional therapies [1]. Although the most frequent driver mutations have been discovered, pathogenesis and mechanisms of melanoma progression have not been completely elucidated so far. Several cellular pathways were described to be activated during melanoma progression, such as rat sarcoma (RAS)/v-raf murine sarcoma viral oncogene homolog B1 (BRAF)/mitogen-activated protein kinases (MAPK) and phosphatidylinositol 3-kinase (PI3K)/AKT, have been reported to promote or downregulate different B-cell lymphoma-2 (Bcl-2) family molecules. Bcl-2 family members are mainly involved in the control of intrinsic apoptotic pathways by regulating mitochondrial integrity and caspases activation. They are expressed in normal melanocytes and are frequently deregulated in melanoma [2]. Previously studies found that both Bcl-2 and Bcl-xL, antiapoptotic members of the Bcl-2 family, were associated with melanoma progression, resistance to apoptosis, and poor prognosis by acting on processes, such as cell proliferation, migration, invasion, and activating crosstalk with the tumor microenvironment [2-4].

Bcl-2-like protein-10 (Bcl2L10), also known as Bcl-B and $\mathrm{NrH}$, is one of the less studied and latest identified proteins belonging to Bcl-2 family [5-7]. It is widely expressed in human tissues and normal plasma cells [8]. Bcl2L10 is also evidenced in tumors from different origins, including breast, prostate, gastric and colorectal adenocarcinomas, as well as non-small cell lung cancer and papillary thyroid carcinoma [8, 9]. Bcl2L10 expression in hematological malignancies, such as multiple myeloma and diffuse large B-cell lymphoma, was also found $[8,10]$. Very recently, high Bcl2L10 expression both in melanoma cell lines and specimens from melanoma patients has been reported [11]. An association of Bcl2L10 expression with poor prognosis in breast, prostate, colorectal, and small cell lung cancer has been also evidenced $[8,12]$. On the contrary, Bcl2L10 correlated with a better outcome in gastric cancer where it works as a tumor suppressor; moreover, hypermethylation of the gene promoter has been found to play an important role in silencing its expression $[8,13,14]$.

Bcl2L10 shows both pro- and anti-apoptotic roles, which are related to cell or tissue context [5, 6, 15-17]. In some cases, Bcl2L10 was even reported not to affect apoptosis of cancer cells, thus indicating that in several models it may not be a regulator of apoptosis [18]. A different effect on apoptosis has been proposed to be related to the endogenous expression level of Bcl2L10 protein [19]. Inhibition of calcium release from the endoplasmic reticulum by Bcl2L10 has been reported as the cause of its anti-apoptotic activity [12]. Very recently, the ability of Bcl2L10 to protect melanoma cells from the cytotoxic effect of different drugs has been demonstrated [11]. The involvement of Bcl2L10 in suppressing autophagic cell death caused by several stimuli, in a beclin-1-dependent manner, has also been reported in gastric and cervical cancer [17, 20, 21].

Polyubiquitination and proteasomal turnover, as well as gene amplification, have been identified as relevant mechanisms dictating Bcl2L10 expression [22-24]. Ubiquitin-dependent proteasomal degradation has been found to affect Bcl2L10 function as regulator of apoptosis, while increased expression of Bcl2L10 by gene amplification has been linked to acquired drug resistance in cancer cells [23, 24]. Bcl2L10 also represents a predictive factor for resistance to azacitidine in myelodysplastic syndromes and acute myeloid leukemia patients [25], and for response to neoadjuvant chemoradiotherapy in locally advanced rectal cancer [9] 
or to death-inducing agents in breast cancer [12]. The analysis of the prevalence of Leucine21Arginine polymorphism of Bcl2L10 in patients previously treated with radiotherapy and/or chemotherapy evidenced that patients with this polymorphic variant show a decreased risk to develop therapy-related myeloid neoplasms and de novo myelodysplastic syndromes [26]. Bcl2L10 has been also reported to participate in tumorigenicity through regulation of invasive/migratory ability of ovarian cancer, as well as angiogenesis and metastatization of hepatocellular carcinoma $[9,13]$. Moreover, as evidenced for other pro-survival Bcl-2 family proteins [27], Bcl2L10 cooperates with Myc to induce leukemogenesis [22].

As at present, there is no evidence about the involvement of Bcl2L10 in melanoma progression, the current study aims at investigating whether Bcl2L10 affects properties known to be associated with tumor aggressiveness, such as in vitro and in vivo tumor growth as well as migration, invasion and capillary-like structure formation [vasculogenic mimicry (VM)].

\section{Materials and methods}

\section{Cell culture}

M14, A375 and WM115 human melanoma cell lines were purchased from American Type Culture Collection (Manassas, VA, USA). SBCL1 human melanoma cell line was provided by Dr. B.C. Giovanella [28]. Patient-derived human melanoma cells ME70, ME10538, ME1007, ME4405 and ME14464 were provided by Dr. A. Anichini [29]. All cell lines were grown in Roswell Park Memorial Institute (RPMI) medium (Euroclone, Milan, Italy) containing $10 \%(v / v)$ fetal bovine serum (FBS), $1 \%$ penicillin/streptomycin and $1 \%$ l-glutamine (Euroclone) in a balanced air humidified incubator with $5 \% \mathrm{CO}_{2}$ and at $37^{\circ} \mathrm{C} . \mathrm{M} 14, \mathrm{~A} 375$, WM115, ME10538, ME14464 carried BRAF mutation, while ME1007 and ME4405 were BRAF wild type.

M14, A375, SBCL1 and ME4405 cell lines were authenticated using high-throughput single nucleotide polymorphisms (SNP)-based assays. Authentication for the other cell lines is ongoing. Experiments were performed with mycoplasma-free cells.

Bcl2L10 stably overexpressing clones were obtained transfecting $1.5 \times 10^{5} \mathrm{M} 14$ cells with empty vector or Bcl2L10 vector expressing the full-length human Bcl2L10 protein fused to the $\mathrm{N}$-terminal Myc epitope tag [11]. Jet-PRime reagents (Polyplus Co., Illkirch, France) were used according to the manufacturer's protocol. Control (M14-C) and Bcl2L10 overexpressing (M14-B) clones were then selected in the presence of $1,200 \mu \mathrm{g} / \mathrm{mL}$ geneticin. For transient transfection, $1.5 \times 10^{5} \mathrm{~A} 375$ cells were transfected with empty or $B c l 2 L 10$ expressing vectors, or with pooled oligonucleotide mix against $B c l 2 L 10$ or scramble target sequences [20 nmol/L si-Bcl2L10 or si-Ctrl (Dharmacon RNA Technologies, siGENOME SMARTpool, Lafayette, CO, USA) using INTERFERin ${ }^{\circledR}$ (Polyplus Co.)] according to the manufacturer's instructions. Control (A375-C), Bcl2L10 overexpressing (A375-B), Bcl2L10 interfered (si-Bcl2L10), and scramble interfered (si-Ctrl) cells were obtained. After $48 \mathrm{~h}$ of transfection, protein expression was evaluated. For urokinase plasminogen activator receptor (UPAR) silencing, $1.5 \times 10^{5} \mathrm{Bcl} 2 \mathrm{~L} 10$ overexpressing M14 cells were transfected with a 20 nmol/L si-uPAR or si-Ctrl (Dharmacon RNA Technologies) using INTERFERin $®$ (Polyplus Co.) according to the manufacturer's instructions. After $48 \mathrm{~h}$ of transfection, protein expression was evaluated.

\section{Intracellular calcium levels determination}

For intracellular calcium levels $\left(\left[\mathrm{Ca}^{2+}\right]_{\mathrm{i}}\right)$ determination, M14-C and M14-B stable clones cultured on 35 $\mathrm{mm}$ dishes were incubated in culture medium containing $3.5 \mu \mathrm{mol} / \mathrm{L} 2$-[6-[bis[2-[(Acetyloxy)methoxy]2-oxoethyl]amino]-5-[2-[2-[bis[2-[(acetyloxy)methoxy]-2-oxoethyl]amino]-5-methylphenoxy]ethoxy]2-benzofuranyl]-5-oxazolecarboxylic acid (acetyloxy)methyl ester (FURA-2-AM, Invitrogen, Carlsbad, California, USA) for $30 \mathrm{~min}$ at $37^{\circ} \mathrm{C}$, and then rinsed with Hank's balanced salt solution (Sigma-Aldrich, St. Louis, Missouri, USA). Dishes were placed into a culture chamber on the support of an inverted fluorescence microscope (Nikon TE2000E, Nikon Instruments, Italy), at $37^{\circ} \mathrm{C}$ connected to a cooled charge-coupled devices camera (12B cascade, Roper Scientific, Ottobrunn, Germany). Random access monochromator was used to illuminate samples alternately at 340 and $380 \mathrm{~nm}$ (Photon Technology International, New Jersey, USA). A $510 \mathrm{~nm}$ emission filter was used to detect emission. Cells were stimulated with $100 \mu \mathrm{m}$ histamine. 
Metafluor ${ }^{\circledR}$ software (Universal Imaging Corporation, Downington PA, USA) was used to acquire images (1 ratio image per s). At the end of each experiment, calibration was obtained by maximally increasing intracellular $\mathrm{Ca}^{2+}$-dependent FURA-2-AM fluorescence with $5 \mu \mathrm{mol} / \mathrm{L}$ ionomycin (ionomycin calcium salt from Streptomyces conglobatus, Sigma) followed by recording minimal fluorescence in a $\mathrm{Ca}^{2+}$-free medium. $\left[\mathrm{Ca}^{2+}\right]_{\mathrm{i}}$ was calculated as previously described [30].

\section{Western blotting analysis and zymography}

Cells were lysed in $10 \mathrm{mmol} / \mathrm{L}$ trisaminomethane hydrochloride buffer $\mathrm{pH} 7.4$ with $2 \%$ sodium dodecyl sulphate (SDS) and fresh protease inhibitors. Protein concentrations were determined by colorimetric assay after extracts sonication for $20 \mathrm{~s}$ (Pierce ${ }^{\mathrm{TM}}$ BCA Protein Assay Kit, Thermo Scientific, Waltham, Massachusetts, USA). The following primary antibodies were used to perform western blotting: Bcl2L10 (\#3869, Cell Signaling, Danvers, MA, USA), p44/42 [extracellular-signal-regulated kinase (ERK)1/2, \#9102, Cell Signaling], phosphorylated p44/42 (ERK1/2, \#9106 L, Cell Signaling) and MMP2 (H-76, sc-10736, Santa Cruz Biotechnology, Santa Cruz, CA). $\beta$-actin (\#A1978, Sigma-Aldrich) and heat shock protein (HSP)72/73 (\#HSP01, Calbiochem, San Diego, CA, USA) were used to check equivalent transfer and loading. Chemiluminescent method (Pierce, Rockford, IL) was used to detect immunostained bands. Image Lab ${ }^{\text {TM }}$ Software (Bio-Rad, Hercules, CA, USA) and ChemiDoc System instrument (Bio-Rad), were used to acquire images, while ImageJ software was used for densitometric evaluation and normalization with relative controls.

Cultured medium (CM) from M14-B and M14-C cells incubated in serum free medium (SFM) for $24 \mathrm{~h}$ was normalized to the number of adherent cells and assayed for gelatinase activity using $7.5 \%$ SDS gels containing gelatin $(0.1 \mathrm{mg} / \mathrm{mL})$ as previously described [31, 32].

In vitro cell migration, invasion, and VM (capillary-like structure formation) assays

Two-dimensional (2D) cell migration and invasion were performed at $37^{\circ} \mathrm{C}$ for $8 \mathrm{~h} \mathrm{using}$ ThinCerts (Greiner Bio-one, Kremsmünster, Austria) containing $8 \mu \mathrm{m}$ pore polycarbonate membrane as previously reported $[4,29,33]$. A total of $5 \times 10^{4}$ cells (M14-C, M14-B, A375-C, A375-B, A375 si-Ctrl, A375 si-Bcl2L10) were seeded in SFM into the upper chamber of ThinCerts. For invasion assay, the ThinCerts were previously coated with basement membrane extract (BME, Cultrex, 8-12 mg/mL, R\&D Systems, Minneapolis, MN, USA) diluted 1:25 with cold SFM, and incubated for $1 \mathrm{~h}$ at $37^{\circ} \mathrm{C}$. The lower well contained medium with $10 \%$ FBS (Hyclone, Thermo Scientific, South Logan, UT) After migration or invasion, a cotton swab was used to remove the cells present in the topside of the membrane. Then, migrating/invading cells were fixed and stained by using differential quick stain kit (Dade Behring, Marburg, Germany). Images were acquired by using a Nikon Eclipse Ts100 microscope and Infinity software and then the migrated/invaded cells were counted. At least five images were acquired for each condition. 2D cell migration and invasion assay were also performed in M14 cells stably overexpressing Bcl2L10 after pharmalogical inhibition of uPAR, using the M25 peptide (YHHLSLGYMYTLN) at $50 \mu \mathrm{mol} / \mathrm{L}$, as previously reported [33]. M25 peptide has been designed to impair the interaction between integrin $\alpha$ chain and uPAR, thus inactivating the subsequent signaling pathway [34, 35]. In this last condition, images were acquired by using the light channel of Bio-Rad ZOE fluorescent cell imager (Bio-Rad Laboratories).

Analysis of VM was performed as previously reported by using 24-well plates [4, 29, 33]: $250 \mu \mathrm{L}$ of BME were dropped onto each well and were allowed to solidify in humidified $5 \% \mathrm{CO}_{2}$ incubator for $1 \mathrm{~h}$ at $37^{\circ} \mathrm{C}$. A total of $1 \times 10^{5} \mathrm{M} 14$ and $1.5 \times 10^{5} \mathrm{~A} 375$ cells were seeded in SFM onto the gelled BME and incubated at $37^{\circ} \mathrm{C}$ for $18 \mathrm{~h}$. Capillary-like structures formation was photographed using Nikon Eclipse Ts100 light microscopy and quantified in 10 sets of images for condition. VM formation was also evaluated in M14 cells stably overexpressing Bcl2L10 after treatment with M25 peptide at $50 \mu \mathrm{mol} / \mathrm{L}$ for $24 \mathrm{~h}$, and acquiring the images by using the light channel of Bio-Rad ZOE fluorescent cell imager (Bio-Rad Laboratories). 


\section{Three-dimensional spheroid invasion assay}

Cultrex 3-D spheroid cell invasion assay (Cultrex \#3500-096-K) was employed for three-dimensional (3D) invasion assay, following the manufacturer's instructions. Spheroids of A375 transfected cells were generated as previously reported by plating 500 cells for $48 \mathrm{~h}$ in 3D culture qualified 96-well plate [36]. Then, spheroids were embedded into the Cultrex spheroid invasion matrix. After $1 \mathrm{~h}$ at $37^{\circ} \mathrm{C}$, SFM with or without broad-spectrum matrix metalloprotease inhibitor, Ilomastat/GM6001 (Cat\# GM6001; Millipore, Burlington, Massachusetts, USA) or uPAR inhibitor, M25 peptide, was added to spheroids wells. Plates were incubated for 7 days, and spheroids were photographed every $24 \mathrm{~h}$ through the use of Bio-Rad ZOE fluorescent cell imager (Bio-Rad). ImageJ software (https://imagej.nih.gov/ij/) was used to measure invasion area. Captured images were converted to 8 bits, and the threshold was set to capture the total structure and calculate the area of the spheroids.

\section{Statistical analysis}

Results are expressed as mean \pm standard deviation (or standard error of the mean where specified) of at least three independent experiments, unless specified. Differences between groups were analyzed with an unpaired two-tailed student's $t$ test with Welch's correction and considered statistically significant for $P<0.05$.

\section{Results}

$B C 12 L 10$ is expressed in human melanoma cells and regulates intracellular level of calcium

We first evaluated the endogenous levels of Bcl2L10 protein in a panel of established and primary human melanoma cells [29]. In agreement with our recent findings [11], Bcl2L10 protein was expressed in all cells tested, although with different extent, thus confirming its expression in melanoma cells (Figure 1A). A barely detectable expression of Bcl2L10 protein was also observed in the transformed melanocytes (Figure S1). By interrogating the Human Protein Atlas database (https://www.proteinatlas.org/), Bcl2L10 protein showed cytoplasmic expression in several normal tissues, most abundantly in liver and kidney. It is noteworthy that looking at data reported by the database for single cell type belonging to "skin", melanocytes are not expressing Bcl2L10 mRNA (Figure S1). Moreover, no detection has been reported in skin specimens by immunohistochemistry (https://www.proteinatlas.org/), differently to what has been reported for other proteins belonging to Bcl-2 family, such as Bcl-2 and Bcl-xL, which are strongly expressed in normal melanocytes [2].

To assess the functional relevance of Bcl2L10 on in vitro melanoma aggression properties, we generated control (M14-C) and Bcl2L10 overexpressing (M14-B) stably clones from M14 melanoma cells (Figure 1B). As Bcl2L10 has been reported to exert its antiapoptotic activity in breast cancer models through inhibition of calcium release from the endoplasmic reticulum [12], we firstly characterized the contribution of Bcl2L10 overexpression to calcium release in melanoma model. To achieve this purpose, both control and Bcl2L10 overexpressing M14 cells were stimulated with histamine, and intracellular calcium was assayed in FURA-2-AM loaded single cells. Bcl2L10 overexpression inhibited calcium signaling respected to control cells (Figure 1C, 1D).

\section{Bcl2L10 promotes in vitro melanoma cell migration, invasion, and VM}

Next, we investigated the role of Bcl2L10 in in vitro proliferation, clonogenic ability and in vivo tumor growth. Although previously published data demonstrated Bcl2L10 ability to affect proliferation of ovarian [18], gastric [19] and hepatocellular [13] carcinoma, we did not observe any effect of Bcl2L10 either on proliferation or clonogenic ability of melanoma cells (Figure S1). Different from the findings evidencing Bcl2L10 as a tumor growth inhibitor in hepatocellular carcinoma [13], and in agreement with our in vitro results obtained both in this paper and in the one previously published [11], in vivo experiments demonstrated that Bcl2L10 does not affect in vivo tumor growth of M14 xenografts (Figure S2). We next evaluated the involvement of Bcl2L10 in several steps during tumor progression, such as cell migration, cell invasion, and VM (Figure 2). To achieve this purpose, we performed in vitro transwell migration and invasion assays, finding that Bcl2L10 overexpression 
induced a significant increase of both cell migratory and invasive capacity of M14 cells (Figure 2B, 2E). The ability of Bcl2L10 to affect in vitro cell migration and invasion was confirmed in A375 cells transiently transfected with Bcl2L10 overexpressing plasmid (A375-B cells) (Figure 2A, 2C, 2F) or with specific small interference RNA smart pool targeting Bcl2L10 (si-Bcl2L10) (Figure 2A, 2D, 2G). Considering that in melanoma, the trans-differentiation of plastic tumor cells into a vasculogenic phenotype provides a tumor with a higher level of autonomy and, therefore, higher aggressiveness, we next explored Bcl2L10's role in $\mathrm{VM}$, and the process in which the vessels formed by tumor cells mimic endothelial cell functions [37]. When compared to control cells, Bcl2L10 overexpressing cells showed enhanced VM, evaluated as percentage of capillary-like structures formed after seeding melanoma cells onto the gelled Cultrex BME (Figure 2H). In accordance with this result, we observed a significant reduction of percentage of capillary-like structures in Bcl2L10-silenced A375 cells respected to control ones (Figure 2I).
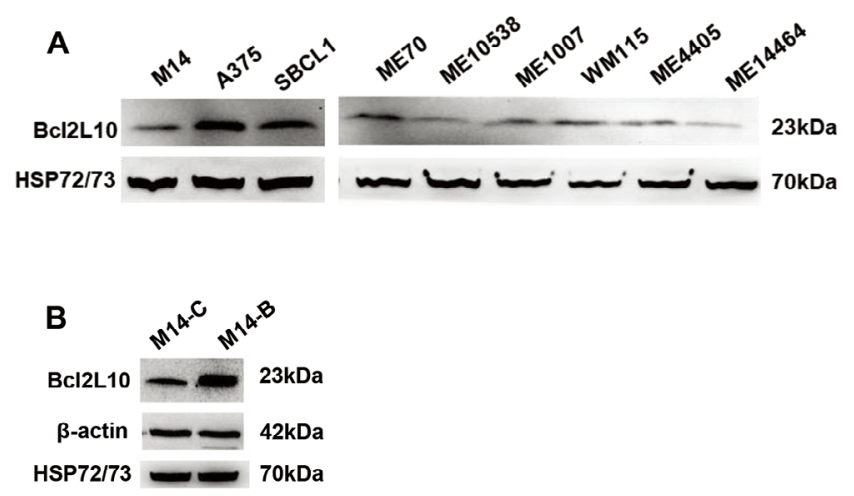

C

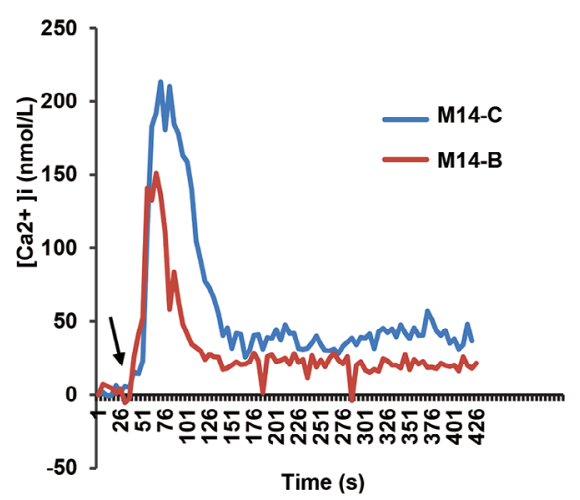

D

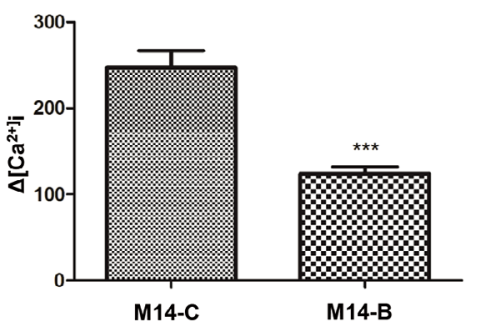

Figure 1. $B C / 2 L 10$ is expressed in human melanoma cells and inhibits intracellular calcium release. (A) Western blot analysis of endogenous Bcl2L10 protein expression in human melanoma cell lines (M14, A375, SBCL1, WM115) and patient-derived melanoma cells (ME70, ME10538, ME1007, ME4405, ME14464). (B) Western blot analysis of Bcl2L10 protein expression in M14 cells stably overexpressing Bc/2L10 (M14-B) obtained by transfecting M14 parental cells with the plasmid expressing the full length human Bcl2L10 protein fused to the N-terminal Myc epitope tag. Empty vector was used to obtain the control clone (M14-C). Reported images are representative of at least two (A) or three (B) independent experiments with similar results. $\beta$-actin and HSP72/73 were used to check equal loading and transfer. (C, D) Live imaging in single FURA-2-AM loaded M14-C and M14-B cells. $\left[\mathrm{Ca}^{2+}\right]_{\mathrm{i}}$ were measured after stimulation with histamine. Calcium levels changes are reported as (C) representative traces and (D) as maximum $\mathrm{Ca}^{2+}$ concentrations in bar charts. Arrow indicates time of histamine addition. Data were expressed as average \pm standard deviation of three independent experiments. $n=30-45$ cells/experiment. Statistical analysis was performed applying $t$ test. ${ }^{* * *} P<0.001$

Bcl2L10 was found to inhibit angiogenesis of hepatocellular carcinoma in vitro and in vivo [13]. Thus, we investigated whether Bcl2L10 was able to affect in vitro and/or in vivo angiogenesis of melanoma models. Human umbilical vein cell line (EA.hy926) seeded on BME and exposed to CM derived from M14 cells overexpressing Bcl2L10 formed a similar number of tubular-like structures when compared to EA.hy926 cells exposed to CM from M14 control cells (Figure S3). In agreement with the in vitro results, matrigel plugs containing CM from Bcl2L10 overexpressing M14 cells injected in C57Bl/6 mice, showed similar haemoglobin content when compared to the matrigel plugs containing CM from control cells (Figure S3). In support of these results, analysis of vascular endothelial growth factor secretion by enzyme-linked immunosorbent 
assay indicated a non-significant induction (1.2 \pm 0.14 -fold) of vascular endothelial growth factor in Bcl2L10 transfectants, respect to control cells $(P=0.18)$. These results indicate that Bcl2L10 contributes to tumor microcirculation by increasing VM but not angiogenesis.

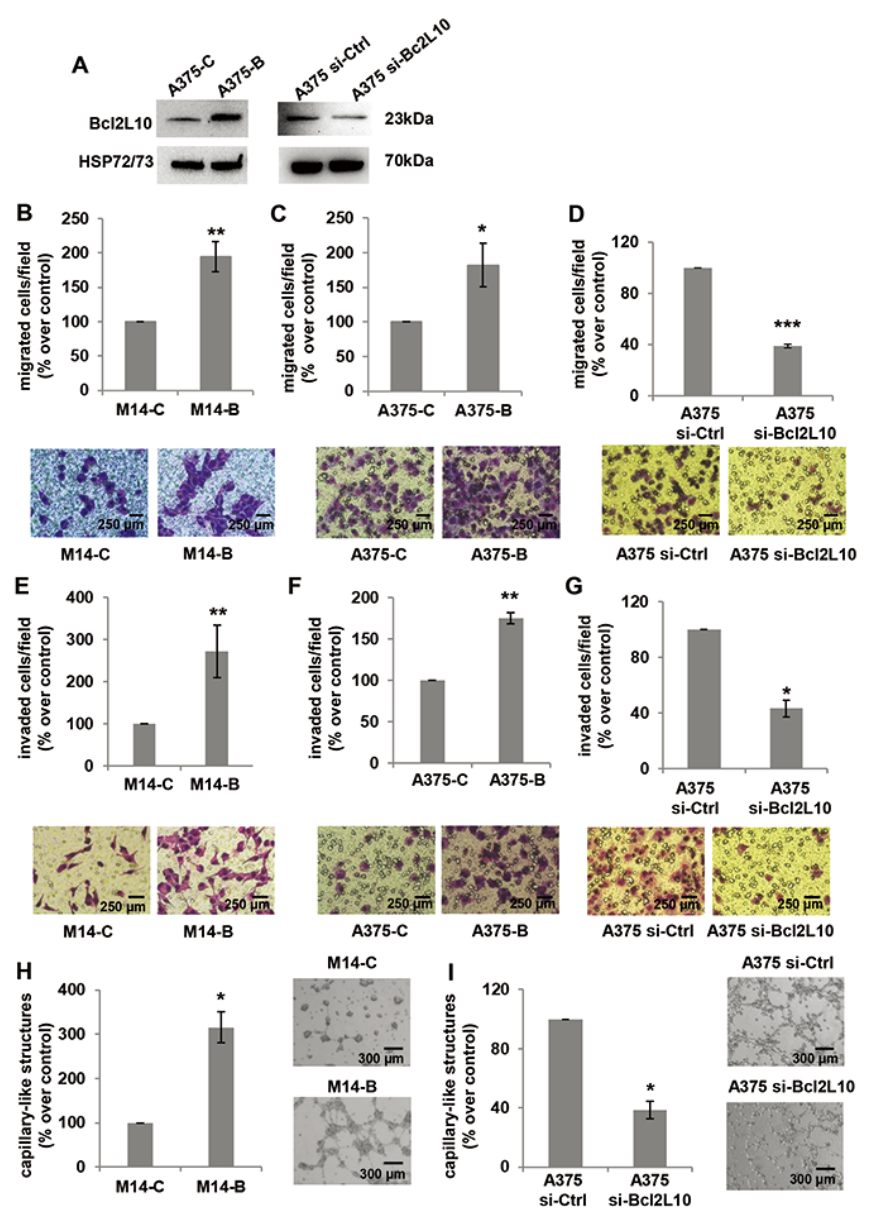

Figure 2. Bcl2L10 promotes in vitro melanoma cell invasion, migration and $\mathrm{VM}$. (A) Western blot analysis of $\mathrm{Bcl} 2 \mathrm{~L} 10$ protein expression in Bcl2L10 overexpressing (A375-B) and control (A375-C) A375 cells, and A375 cells transfected with si-Bcl2L10 (A375 si-Bcl2L10) or si-Ctrl (A375 si-Ctrl). Representative images of three independent experiments with similar results. HSP72/73 was employed to check equal loading and transfer. (B-D) Bar charts and representative images of in vitro cell migration of (B) M14 cells stably overexpressing Bcl2L10 protein (M14-B) and relative control (M14-C), (C) A375-C and A375-B cells, and (D) A375 si-Bcl2L10 or A375 si-Ctrl. (E-G) Bar charts and representative images of in vitro cell invasion of (E) M14-C and M14-B cells, (F) A375-C and A375-B cells, and (G) A375 si-Bcl2L10 or A375 si-Ctrl. Each condition was analyzed in technical duplicate in two $(D, F, G)$ or three $(B, C, E)$ independent experiments. $(B-G)$ Data are reported as the percentage of migrated or invaded cells respected to control. $(\mathrm{H}, \mathrm{I})$ Quantification and representative images of capillary-like structure formation in $(\mathrm{H}) \mathrm{M} 14-\mathrm{B}$ and M14-C cells and (I) in A375 si-Bcl2L10 and A375 si-Ctrl cells. Each condition was analyzed in technical duplicate in three $(\mathrm{H})$ or two $(\mathrm{I})$ independent experiments. Values are expressed as percentage of capillary-like structures formed in M14-B or A375 si-Bcl2L10 cells versus relative controls. (B-I) Data were expressed as mean \pm standard deviation. Scale bars have been reported. Statistical analysis was performed applying unpaired two-tailed student's $t$ test with Welch's correction. ${ }^{*} P<0.05$; ${ }^{* *} P<0.01$; ${ }^{* \star *} P<0.001$

\section{Bcl2L10 expression affects pathways involved in cell migration, invasion, and VM}

As several intracellular pathways, such as the ERK1/2 pathway, are known to be actively involved in cell migration and invasion, we examined the effect of Bcl2L10 modulation on phosphorylation of ERK1/2 (p42/44 MAPK). Overexpressing Bcl2L10 in M14 (Figure 3A) and A375 (Figure 3B) cells increased levels of p42/44 ERK1/2 phosphorylation, while after Bcl2L10 silencing in A375 cells, a significant reduction of phosphorylated p42/44 ERK1/2 was observed (Figure 3C), indicating that this pathway is affected by Bcl2L10 modulation. Next, we evaluated whether Bcl2L10 was able to affect the activity of matrix metalloproteinases MMP2 and MMP9, which we previously demonstrated to be modulated by Bcl-2 and Bcl-xL in melanoma [4,31]. These two zinc dependent proteases play a major role in proteolytic degradation of extracellular matrix components and promote tumor invasion and metastasis [38]. Bcl2L10 overexpressing M14 and A375 cells increased the level of activated MMP2 protein respected 
to controls (Figure 4A, 4B). The Bcl2L10-dependent cleavage of MMP2 protein was confirmed in A375 cells after Bcl2L10 silencing (Figure 4C). In accordance with these results, increased enzymatic activity of MMP2, evaluated by gelatin zymography, was observed in CM from Bcl2L10 overexpressing M14 cells when compared to CM from control ones (Figure 4D). This experiment also allowed appreciating the concomitant activation of the secreted MMP9 in CM from Bcl2L10 overexpressing M14 cells respected to control ones.
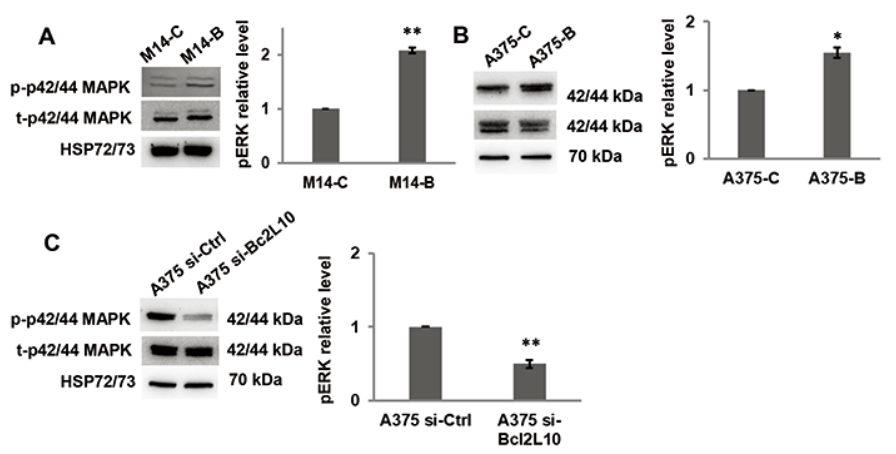

Figure 3. Bcl2L10 promotes ERK phosphorylation. (A-C) Western blot analysis of total ( $t$ ) and phosphorylated (p) p44/42 MAPK (ERK1/2) and relative densitometric analysis of pERK in (A) M14-B and M14-C cells, (B) A375-C and A375-B cells, and (C) A375 si-BCl2L10 and A375 si-Ctrl cells. Reported images are representative of three independent experiments with similar results. HSP72/73 was employed to check equal loading and transfer. For densitometric analysis, the relative levels of proteins were expressed in the histograms as fold changes respected to relative control after normalization. Data were expressed as mean \pm standard deviation. Statistical analysis was performed applying unpaired two-tailed student's $t$ test with Welch's correction. ${ }^{*} P<0.05 ;{ }^{*} P<0.01$

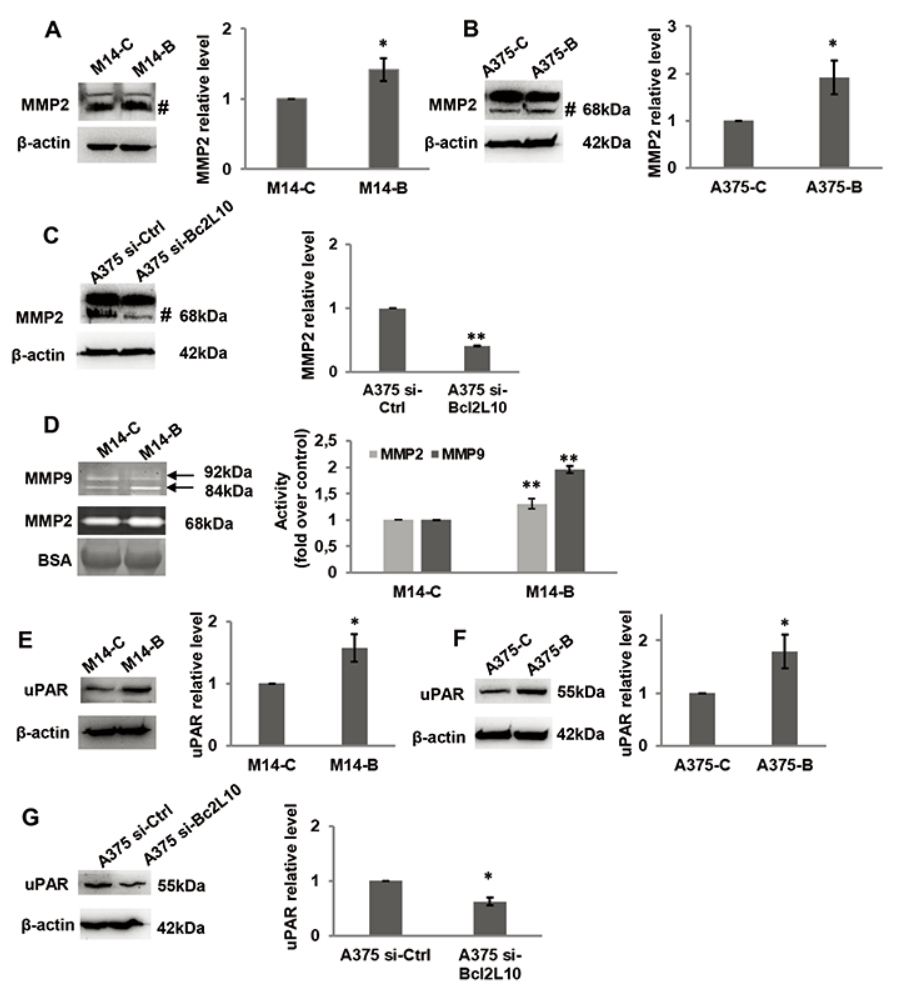

Figure 4. Bcl2L10 promotes metalloproteinases activities and increases uPAR level. (A-C) Western blot analysis and relative densitometric analysis of MMP2 protein expression in protein extracts of (A) M14 cells stably overexpressing Bc/2L10 (M14-B) and relative control (M14-C), and in (B) A375 cells transfected with empty (A375-C) or Bc/2L10 (A375-B) expressing vectors, or (C) with pooled oligonucleotide mix against Bcl2L10 (A375 si-Bcl2L10) or scramble target (A375 si-Ctrl). Active form of MMP2, $68 \mathrm{kDa}$, is indicated $(\#)$. (D) Gelatinase activity and relative densitometric analysis of metalloproteinases MMP2 and MMP9 enzymatic activity of CM from M14-B and M14-C cells incubated in serum free medium for $24 \mathrm{~h}$. Active form of MMP2, $68 \mathrm{kDa}$, and pro- and active form of MMP9, $92 \mathrm{kDa}$ and $84 \mathrm{kDa}$ respectively, are indicated. CM were also analyzed by SDS-polyacrylamide gel electrophoresis, which is transferred to nitrocellulose membrane and bovine serum albumin signal (BSA) was visualized using ponceau staining. (A-D) Representative images of three independent experiments with similar results are shown. (A-C) $\beta$-actin primary antibody was used as loading and transferring control. For densitometric analysis, the relative levels of proteins were expressed in the histograms as fold changes respected to relative control after normalization. Data were expressed as mean \pm standard deviation. Statistical analysis was performed applying unpaired two-tailed student's $t$ test with Welch's correction. ${ }^{*} P<0.05 ;{ }^{* *} P<0.01$. BSA: bovine serum albumin signal 
Further, we evaluated whether Bcl2L10 was able to affect the expression of uPAR, a key regulatory molecule of migration, invasion, and VM [39]. As shown by western blot analysis, we observed an increased level of uPAR protein in Bcl2L10 overexpressing M14 (Figure 4E) and A375 (Figure 4F) cells, while a reduced level of uPAR was detected upon Bcl2L10 silencing in A375 cells (Figure 4G).

We also assessed the invasive capability in a 3D cell culture system using collagen matrix-embedded tumor spheroids derived from A375 cells (Figure 5). Bcl2L10 transiently overexpressing A375 cells (A375-B) enhanced invasion into the surrounding matrix and radial out-growth compared to control cells (A375-C), which is impaired by both Ilomastat/GM6001, a synthetic pan-inhibitor of matrix metalloproteinases, and M25, a synthetic peptide impairing uPAR functions [33, 35, 40]. M25 application has already validated both by other and us to study the biological role of uPAR in vitro 2D cell migration/invasion, VM, and 3D invasion assay [33-35].

A
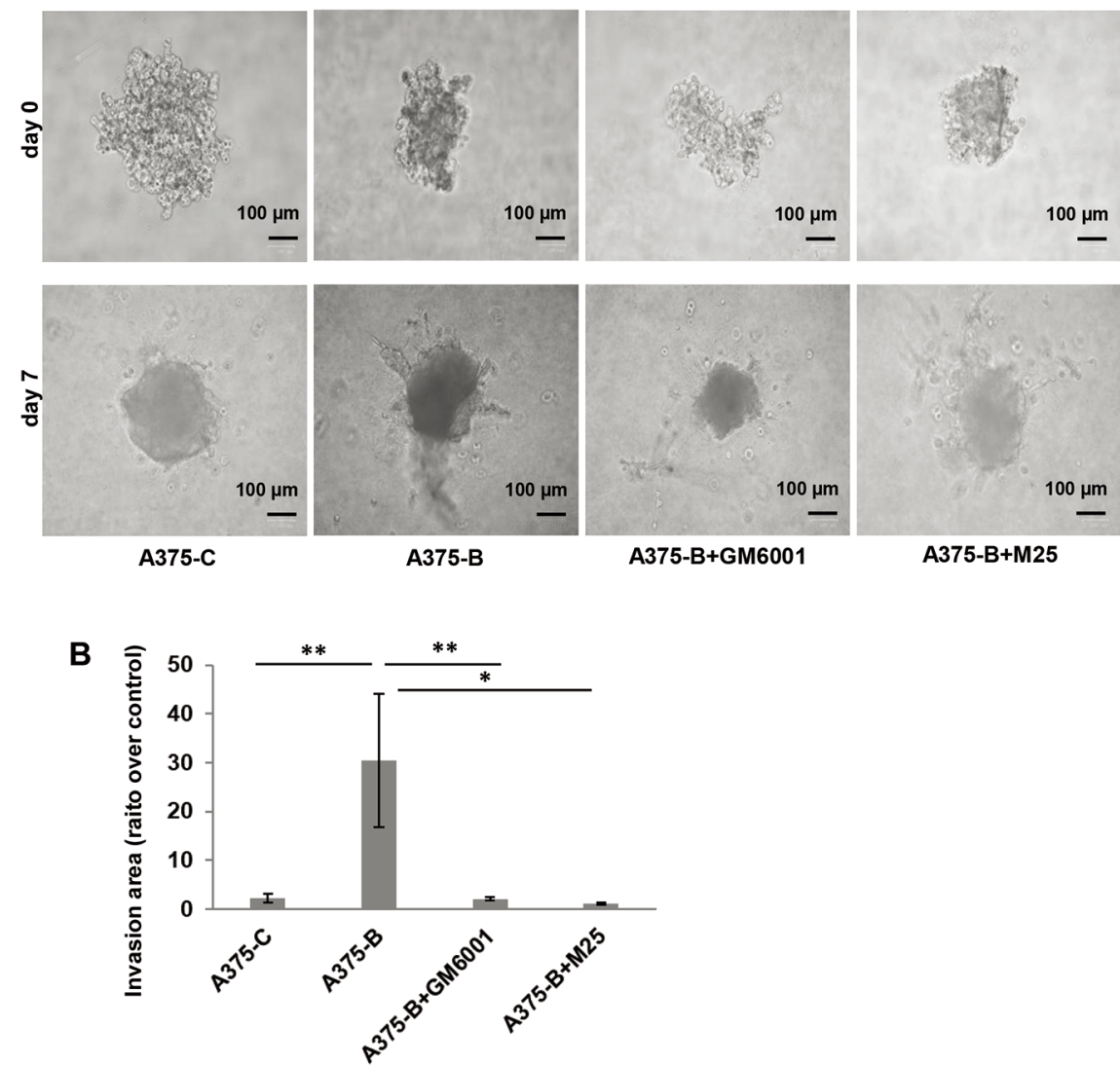

Figure 5. Bcl2L10 enhances the ability of melanoma cells to invade. (A) Representative images of $3 D$ spheroid invasion assays using $A 375$ cells transfected with empty (A375-C) or Bc/2L10 (A375-B) expressing vectors over 7 days following the addition of invasion matrix, and A375-B cells treated with matrix metalloproteinases (MMPs) inhibitor (llomastat/GM6001) or uPAR inhibitor (peptide M25). (B) Histograms, quantification of 3D invasion relative to invasion area. Data were expressed as mean \pm standard error of the mean. Statistical analysis was performed applying one-way analysis of variance, Tukey post-hoc analysis. ${ }^{*} P<0.05 ;{ }^{*} P<0.01$

Our data obtained in 3D model demonstrate that both MMPs and uPAR mediate the effect of Bcl2L10 in cellular invasion.

To more deeply characterize the relevance of UPAR in the ability of Bcl2L10 to affect in vitro properties associated with melanoma aggressiveness, cell migration/invasion and VM were analyzed after uPAR downregulation in M14 cells stably overexpressing Bcl2L10 by using a specific small interference RNA smart pool (si-uPAR), which is able to reduce both uPAR mRNA and protein expression. As expected, uPAR silencing did not affect both Bcl2L10 protein and mRNA expression (Figure 6A, Figure S4). Downregulation of uPAR levels strongly reduced in vitro cell migration/invasion and VM of Bcl2L10 overexpressing melanoma 
cells (Figure 6B-D). In order to confirm these results, we performed in vitro cell migration, cell invasion, and VM assays after pharmacological inhibition of uPAR with M25 peptide. As shown in Figure S5, M25 peptide significantly reduced cell migration, invasion, and capillary-like structure formation. These results demonstrate that uPAR plays a role in Bcl2L10-dependent promotion of cell migration/invasion, and VM.
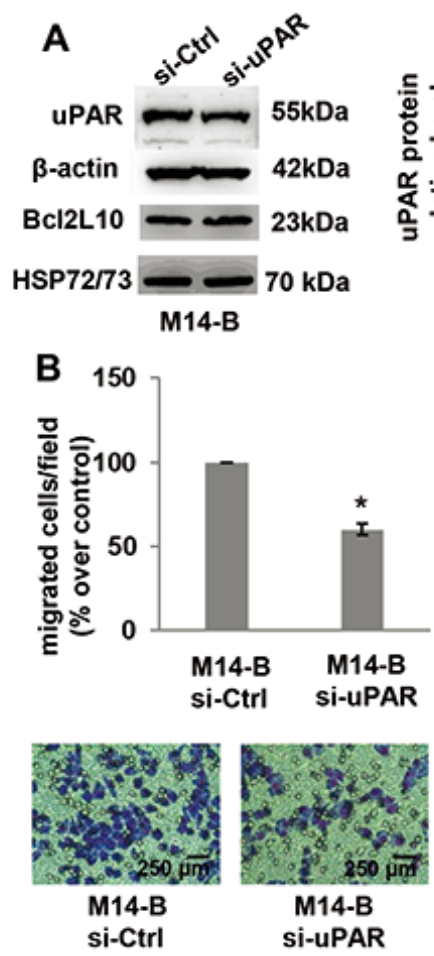

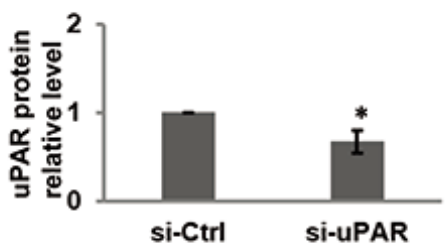

C

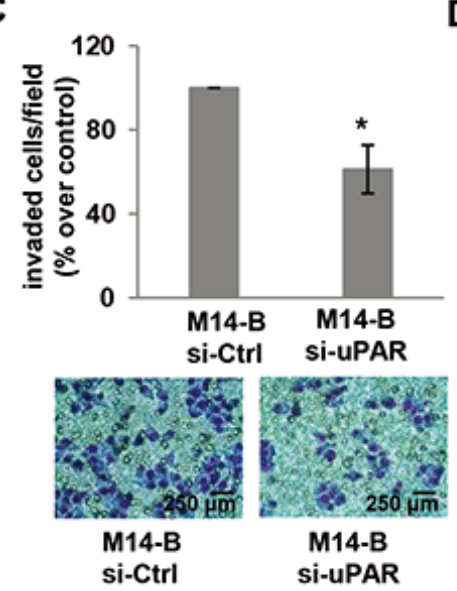

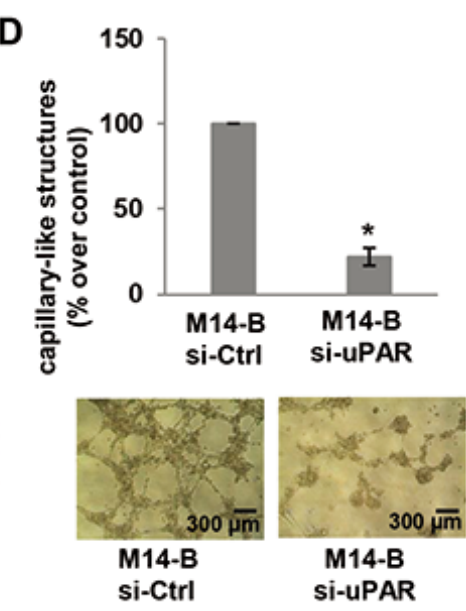

Figure 6. Bcl2L10 promotes migration, invasion, and VM of melanoma cells through uPAR. (A) Western blot analysis of uPAR and Bcl2L10 and densitometric analysis of UPAR protein expression in Bcl2L10 overexpressing M14 clone (M14-B) transfected with siRNA oligonucleotides against uPAR (si-uPAR) or scramble (si-Ctrl) target sequences. Reported images are representative of three independent experiments with similar results. $\beta$-actin and HSP72/73 were employed to check equal loading and transfer. For densitometric analysis, protein levels were quantified by using ImageJ software and the relative levels of proteins were expressed in the histograms as ratio changes respect to relative control after normalization. Data were expressed as mean \pm standard deviation. Statistical analysis was performed applying corrected $t$ test. (B-D) Quantification and representative images of in vitro cell (B) migration, $(C)$ invasion, and (D) capillary-like structure formation in M14-B cells transfected with siRNA oligonucleotides against UPAR (si-uPAR) or scramble (si-Ctrl) target sequences. Values are expressed as a percentage of migrated/invaded cells or capillary-like structure formed respect to control. (B-D) Each condition was analyzed in duplicate in three (C) or two (B, D) independent experiments. Data were expressed as mean \pm standard deviation. Scale bars have been reported. Statistical analysis was performed applying unpaired two-tailed student's $t$ test with Welch's correction. * $P<0.05$

\section{Discussion}

Here we demonstrated the ability of Bcl2L10 to promote a more aggressive in vitro phenotype in melanoma models. In particular, in accordance with data demonstrating Bcl2L10 expression in human melanoma cells and melanoma patient specimens [11], we confirmed the expression of endogenous Bcl2L10 protein in a panel of melanoma cells. Of note, by using human melanoma cells stably overexpressing Bcl2L1O or transiently transfected for loss/gain function of Bcl2L10, we evidenced that Bcl2L10 is an inducer of in vitro cell invasion/migration and VM, similarly to what was observed for other anti-apoptotic proteins, such as Bcl-2 and Bcl-xL $[4,31]$. The ability of Bcl2L10 to promote in vitro cell invasion was demonstrated by using both 2D assays and 3D culture of tumor spheroids, the latter recapitulating in vivo human solid tumors.

By western blotting and zymography assays, we showed that MMP2 and MMP9 protein activities were increased by Bcl2L10. MMP family plays a relevant role in the degradation of extracellular matrix components and basement membrane [38]. Among the MMPs, MMP2 and MMP9 represent the most important proteins in metastasis [41]. The use of a MMPs inhibitor in 3D spheroid invasion assay reduced invasiveness of melanoma cells overexpressing Bcl2L10 protein, pointing out the role of MMPs in mediating Bcl2L10 function. 
In addition to MMPs, uPAR also acts as a proteolytic degrader of extracellular matrix [42]. Interestingly, we observed a remarkable reduction of radial out-growth of Bcl2L10 overexpressing cells in 3D culture system after pharmacological inhibition of uPAR. In addition, a significant reduction of both cell migration and invasion in 2D assays after both UPAR mRNA downregulation using RNA interference and pharmacological blocking has been observed. These data indicate that, in addition to MMPs, uPAR also could play a role in promoting Bcl2L10-dependent migration and invasion functions. Several studies support the relevance of the plasminogen activator system in melanoma: urokinase plasminogen expression correlates with the metastatic potential of melanoma models, and the expression of urokinase plasminogen and its cognate receptor uPAR are increased in late-stage melanocytic tumors [43]. Other studies support a direct involvement of uPAR in the melanoma progression: hypoxia promotes lymph-node metastasis in human melanoma xenografts by upregulating UPAR [44]. Moreover, inhibition of UPAR through RNA interfering reduces tumor growth and induces pro-apoptotic effects in melanoma models with acquired resistance to target therapy [45, 46].

In this study we also observed that the modulation of Bcl2L10 also induces phosphorylation of ERK1/2, a relevant pathway in melanoma pathobiology. In fact, high ERK1/2 phosphorylation levels have been detected in clinical melanoma metastases and various melanoma cell lines [47], and combination of BRAF and mitogen-activated protein kinase inhibition is the elective treatment in patients with advanced BRAF-mutant melanoma.

Moreover, we evidenced the ability of Bcl2L10 to promote the formation of VM, which is a phenomenon indicating the de novo formation of vasculogenic-like networks by aggressive tumor cells, and correlating with high tumor grade, short survival, invasion, and metastasis [48, 49]. Of note, in this work, the formation of capillary-like structures was impaired by both genetic and pharmacological targeting of uPAR, thus demonstrating the role played by uPAR in Bcl2L10-dependent VM. Several molecular mechanisms and signal pathways are responsible of VM induction and formation [48, 50]. Among the others, increased activation of ERK1/2 and MMP2, as well as upregulation of MMP14, have been reported in melanoma cells [51]. Moreover, positive correlation of UPAR expression with VM formation, metastasis, and poor prognosis in different aggressive cancers has been reported [49]. In this content, we previously evidenced that inhibition of UPAR expression, by genetic approach in melanoma cells, decreased the in vitro VM formation induced by miR-378a-5p. More recently, it has been reported that uPAR targeting by M25 peptide could impair capillary-like formation in drug-resistant melanoma cells [34]. Moreover, we demonstrated that Bcl-xL was also able to induce VM in both in vitro and in vivo model of melanoma [4].

Although we demonstrated a relevant involvement of Bcl2L10 in promoting migration, invasion and formation of capillary-like structures, we did not observe any substantial effect of Bcl2L10 in the regulation of in vitro clonogenic ability and, in accordance with our previously published data [11], of cell proliferation. Unlike our results, Bcl2L10 knockdown has been reported to promote the proliferation of ovarian [18] and gastric [19] cancer cells. Moreover, ectopic expression of Bcl2L10 in hepatocellular carcinoma models suppressed both cell viability and colony formation [13]. In agreement with our in vitro results, we found that in vivo tumor growth was not affected by Bcl2L10. On the contrary, overexpression of Bcl2L1O inhibited the growth of hepatocellular carcinoma xenografts and reduced experimental lung metastasis [13]. In addition, forced expression of Bcl2L1O in hepatocellular carcinoma model reduced in vitro and in vivo angiogenesis [13], while we did not observe significant differences in vitro and in vivo angiogenesis assay after Bcl2L10 forced expression. On the basis of these evidence, we can suggest that the effect of Bcl2L10 is strictly depended on the tumor histotype.

We have previously demonstrated that Bcl2L10 is an anti-apoptotic protein in melanoma, where it is able to protect cells from the cytotoxic effect of different drugs, such as cisplatin, dacarbazine, ABT-737 (a pan Bcl-2 inhibitor) and PLX-4032 (a BRAF inhibitor, administered alone or in combination) [11]. In accordance with our previous results demonstrating the anti-apoptotic activity of Bcl2L10 [11], here we found a decreased $\left[\mathrm{Ca}^{2+}\right]_{\mathrm{i}}$ in melanoma cells overexpressing Bcl2L10. These results agree with those obtained in breast cancer, where Bcl2L10 has been reported to promote its anti-apoptotic function by negatively regulating the release of calcium from the endoplasmic reticulum through the interaction of the Bcl-2 homology (BH) 4 domain with 
inositol-1,4,5-trisphosphate receptor [12]. In addition to proliferation and cell death, intracellular calcium dynamics regulate many cellular processes including cytoskeleton remodeling and cell migration [52].

Bcl-2 proteins have been reported to be localized at the mitochondria-endoplasmic reticulum interface and to interact with key channels or receptors on both endoplasmic reticulum and mitochondrial membranes. Thus, the role of $\mathrm{Bcl}-2$ proteins in regulating intracellular and mitochondrial $\mathrm{Ca}^{2+}$ homeostasis, and subsequently to several other processes such as cell migration, independently of apoptosis, is emerging [53]. Increased intracellular $\mathrm{Ca}^{2+}$ levels have been described to be associated to the anti-migration effects of alkaloid compounds in liver cancer cells [54]. Based on this consideration, we can hypothesize that the increased migration properties observed in melanoma cells overexpressing Bcl $2 L 10$ could also be linked to the reduced $\left[\mathrm{Ca}^{2+}\right]_{\mathrm{i}}$ detected in the same cells.

Due to their multiple functions in cancer, Bcl-2 family proteins have become interesting targets for anti-cancer drugs [55]. The recent approval for clinical practice of venetoclax, a BH3 mimetic specific for Bcl-2, corroborated the clinical relevance of using anti-apoptotic proteins as therapeutic targets, not only for hematologic malignancies but also for solid tumors. In fact, numerous clinical trials are ongoing to evaluate the effects of specific anti-apoptotic Bcl-2 proteins or pan inhibitors as single agents or in combination therapy in solid tumors [55]. Different factors contribute to cellular responsiveness to BH3 mimetic drugs. Among these factors, the differential "addiction" to anti-apoptotic (mainly Bcl-2, Bcl-xL or Mcl-1) proteins has been showed. The concept of "addiction" has been introduced to explain different survival of tumor cells depending on the expression levels of these proteins $[56,57]$. Taking into account of our results evidencing not only the anti-apoptotic function of Bcl2L10 but also its role in promoting in vitro melanoma cell migration, invasion, and VM through the involvement of MMPs and UPAR, we can suggest that Bcl2L10 should be considered in the evaluation of novel anti-apoptotic Bcl-2 protein inhibitors and could represent a potential target for the treatment of melanoma.

\section{Abbreviations}

$\left[\mathrm{Ca}^{2+}\right]_{\mathrm{i}}$ : intracellular calcium levels

2D: two-dimensional

3D: three-dimensional

Bcl-2: B-cell lymphoma-2

Bcl2L10: B-cell lymphoma-2-like protein-10

BH: B-cell lymphoma-2 homology domain

BME: basement membrane extract

BRAF: v-raf murine sarcoma viral oncogene homolog B1

CM: cultured medium

ERK: extracellular-signal-regulated kinase

FURA-2-AM: 2-[6-[bis[2-[(Acetyloxy)methoxy]-2-oxoethyl]amino]-5-[2-[2-[bis[2-[(acetyloxy)methoxy]-2oxoethyl]amino]-5-methylphenoxy]ethoxy]-2-benzofuranyl]-5-oxazolecarboxylic acid (acetyloxy)methyl ester HSP: heat shock protein

MAPK: mitogen-activated protein kinases

MMPs: matrix metalloproteinases

SDS: sodium dodecyl sulphate

SFM: serum free medium

UPAR: urokinase plasminogen activator receptor

VM: vasculogenic mimicry 


\section{Supplementary materials}

The supplementary material for this article is available at: https://www.explorationpub.com/uploads/ Article/file/100268_sup_1.pdf.

\section{Declarations}

Acknowledgments

We thank Dr. Adele Petricca for preparation of the manuscript.

\section{Author contributions}

DDB conceived and designed the study, discussed results and wrote the manuscript; SD conceived and designed the study, performed in vitro experiments, discussed results and wrote the manuscript; MDM performed in vitro and in vivo angiogenesis assays and discussed results; EV, I Manni and CN performed in vivo experiments and discussed results; $\mathrm{AD}$ and $\mathrm{AF}$ determined and discussed the results about intracellular calcium levels; I Masi and LR performed and discussed 3D spheroid invasion assay; MVC and PLB provided some reagents; MJQ performed in vitro experiments; MZ and CC discussed results. All authors contributed to manuscript revision, read, and approved the submitted version.

\section{Conflicts of interest}

The authors declare that there are no conflicts of interest.

\section{Ethical approval}

Not applicable.

\section{Consent to participate}

Not applicable.

\section{Consent to publication}

Not applicable.

Availability of data and materials

Not applicable.

\section{Funding}

The research leading to these results has received funding from AIRC under IG 2020: ID. 24315 project-P.I. Donatella Del Bufalo; ID. 21372 project-P.I. Laura Rosanò. Marta Di Martile was supported by a FIRC-AIRC fellowship for Italy, Project Code: 24057. The funder AIRC had no role in study design, data collection and analysis, decision to publish, or preparation of the manuscript.

\section{Copyright}

(C) The Author(s) 2022.

\section{References}

1. Jenkins RW, Fisher DE. Treatment of advanced melanoma in 2020 and beyond. J Invest Dermatol. 2021;141:23-31.

2. Trisciuoglio D, Del Bufalo D. New insights into the roles of antiapoptotic members of the Bcl-2 family in melanoma progression and therapy. Drug Discov Today. 2021;26:1126-35.

3. Trisciuoglio D, Desideri M, Ciuffreda L, Mottolese M, Ribatti D, Vacca A, et al. Bcl-2 overexpression in melanoma cells increases tumor progression-associated properties and in vivo tumor growth. J Cell Physiol. 2005;205:414-21. 
4. Trisciuoglio D, Tupone MG, Desideri M, Di Martile M, Gabellini C, Buglioni S, et al. BCL- $\mathrm{X}_{\mathrm{L}}$ overexpression promotes tumor progression-associated properties. Cell Death Dis. 2017;8:3216.

5. Inohara N, Gourley TS, Carrio R, Muniz M, Merino J, Garcia I, et al. Diva, a Bcl-2 homologue that binds directly to Apaf-1 and induces BH3-independent cell death. J Biol Chem. 1998;273:32479-86.

6. Song Q Kuang Y, Dixit VM, Vincenz C. Boo, a novel negative regulator of cell death, interacts with Apaf-1. EMBO J. 1999;18:167-78.

7. Zhai D, Ke N, Zhang H, Ladror U, Joseph M, Eichinger A, et al. Characterization of the anti-apoptotic mechanism of Bcl-B. Biochem J. 2003;376:229-36.

8. Krajewska M, Kitada S, Winter JN, Variakojis D, Lichtenstein A, Zhai D, et al. Bcl-B expression in human epithelial and nonepithelial malignancies. Clin Cancer Res. 2008;14:3011-21.

9. Lee IH, Kang K, Kang BW, Lee SJ, Bae WK, Hwang JE, et al. Genetic variations using whole-exome sequencing might predict response for neoadjuvant chemoradiotherapy in locally advanced rectal cancer. Med Oncol. 2018;35:145.

10. Hamouda MA, Jacquel A, Robert G, Puissant A, Richez V, Cassel R, et al. BCL-B (BCL2L10) is overexpressed in patients suffering from multiple myeloma (MM) and drives an MM-like disease in transgenic mice. J Exp Med. 2016;213:1705-22.

11. Quezada MJ, Picco ME, Villanueva MB, Castro MV, Barbero G, Fernandez NB, et al. BCL2L10 is overexpressed in melanoma downstream of STAT3 and promotes Cisplatin and ABT-737 resistance. Cancers (Basel). 2021;13:78.

12. Nougarede A, Popgeorgiev N, Kassem L, Omarjee S, Borel S, Mikaelian I, et al. Breast cancer targeting through inhibition of the endoplasmic reticulum-based apoptosis regulator Nrh/BCL2L10. Cancer Res. 2018;78:1404-17.

13. Bai Y, Wang J, Han J, Xie XL, Ji CG, Yin J, et al. BCL2L10 inhibits growth and metastasis of hepatocellular carcinoma both in vitro and in vivo. Mol Carcinog. 2017;56:1137-49.

14. Mikata R, Fukai K, Imazeki F, Arai M, Fujiwara K, Yonemitsu Y, et al. BCL2L10 is frequently silenced by promoter hypermethylation in gastric cancer. Oncol Rep. 2010;23:1701-8.

15. Kim JH, Yoon S, Won M, Sim SH, Ko JJ, Han S, et al. HIP1R interacts with a member of Bcl-2 family, BCL2L10, and induces BAK-dependent cell death. Cell Physiol Biochem. 2009;23:43-52.

16. Beverly LJ, Varmus HE. MYC-induced myeloid leukemogenesis is accelerated by all six members of the antiapoptotic BCL family. Oncogene. 2009;28:1274-9.

17. Robert G, Gastaldi C, Puissant A, Hamouda A, Jacquel A, Dufies M, et al. The anti-apoptotic Bcl-B protein inhibits BECN1-dependent autophagic cell death. Autophagy. 2012;8:637-49.

18. Lee SY, Kwon J, Woo JH, Kim KH, Lee KA. Bcl2l10 mediates the proliferation, invasion and migration of ovarian cancer cells. Int J Oncol. 2020;56:618-29.

19. Xu JD, Cao XX, Long ZW, Liu XP, Furuya T, Xu JW, et al. BCL2L10 protein regulates apoptosis/proliferation through differential pathways in gastric cancer cells. J Pathol. 2011;223:400-9.

20. Qiu DM, Wang GL, Chen L, Xu YY, He S, Cao XL, et al. The expression of beclin-1, an autophagic gene, in hepatocellular carcinoma associated with clinical pathological and prognostic significance. BMC Cancer. 2014;14:327.

21. He J, Deng L, Liu H, Chen T, Chen S, Xia S, et al. BCL2L10/BECN1 modulates hepatoma cells autophagy by regulating PI3K/AKT signaling pathway. Aging (Albany NY). 2019;11:350-70.

22. Beverly LJ, Lockwood WW, Shah PP, Erdjument-Bromage H, Varmus H. Ubiquitination, localization, and stability of an anti-apoptotic BCL2-like protein, BCL2L10/BCLb, are regulated by Ubiquilin1. Proc Natl Acad Sci U S A. 2012;109:E119-26.

23. van de Kooij B, Rooswinkel RW, Kok F, Herrebout M, de Vries E, Paauwe M, et al. Polyubiquitination and proteasomal turnover controls the anti-apoptotic activity of Bcl-B. Oncogene. 2013;32:5439-48. 
24. Yasui K, Mihara S, Zhao C, Okamoto H, Saito-Ohara F, Tomida A, et al. Alteration in copy numbers of genes as a mechanism for acquired drug resistance. Cancer Res. 2004;64:1403-10.

25. Cluzeau T, Robert G, Mounier N, Karsenti JM, Dufies M, Puissant A, et al. BCL2L10 is a predictive factor for resistance to azacitidine in MDS and AML patients. Oncotarget. 2012;3:490-501.

26. Fabiani E, Fianchi L, Falconi G, Boncompagni R, Criscuolo M, Guidi F, et al. The BCL2L10 Leu21Arg variant and risk of therapy-related myeloid neoplasms and de novo myelodysplastic syndromes. Leuk Lymphoma. 2014;55:1538-43.

27. Swanson PJ, Kuslak SL, Fang W, Tze L, Gaffney P, Selby S, et al. Fatal acute lymphoblastic leukemia in mice transgenic for B cell-restricted bcl-xL and c-myc. J Immunol. 2004;172:6684-91.

28. Verschraegen CF, Mendoza JT, Kozielski AJ, Giovanella BC. Modulation of the response to chemotherapy in a human melanoma clone by the site of growth in the nude mouse. Anticancer Res. 1995;15:9-11.

29. D’Aguanno S, Valentini E, Tupone MG, Desideri M, Di Martile M, Spagnuolo M, et al. Semaphorin 5A drives melanoma progression: role of Bcl-2, miR-204 and c-Myb. J Exp Clin Cancer Res. 2018;37:278.

30. Grynkiewicz G, Poenie M, Tsien RY. A new generation of $\mathrm{Ca}^{2+}$ indicators with greatly improved fluorescence properties. J Biol Chem. 1985;260:3440-50.

31. Gabellini C, De Luca T, Trisciuoglio D, Desideri M, Di Martile M, Passeri D, et al. BH4 domain of bcl-2 protein is required for its proangiogenic function under hypoxic condition. Carcinogenesis. 2013;34:2558-67.

32. Chellini L, Caprara V, Spadaro F, Sestito R, Bagnato A, Rosanò L. Regulation of extracellular matrix degradation and metastatic spread by IQGAP1 through endothelin-1 receptor signalling in ovarian cancer. Matrix Biol. 2019;81:17-33.

33. Tupone MG, D’Aguanno S, Di Martile M, Valentini E, Desideri M, Trisciuoglio D, et al. microRNA-378a-5p is a novel positive regulator of melanoma progression. Oncogenesis. 2020;9:22.

34. Andreucci E, Laurenzana A, Peppicelli S, Biagioni A, Margheri F, Ruzzolini J, et al. uPAR controls vasculogenic mimicry ability expressed by drug-resistant melanoma cells. Oncol Res. 2021; [Epub ahead of print].

35. Laurenzana A, Margheri F, Biagioni A, Chillà A, Pimpinelli N, Ruzzolini J, et al. EGFR/uPAR interaction as druggable target to overcome vemurafenib acquired resistance in melanoma cells. EBioMedicine. 2019;39:194-206.

36. Masi I, Caprara V, Spadaro F, Chellini L, Sestito R, Zancla A, et al. Endothelin-1 drives invadopodia and interaction with mesothelial cells through ILK. Cell Rep. 2021;34:108800.

37. Folberg R, Hendrix MJ, Maniotis AJ. Vasculogenic mimicry and tumor angiogenesis. Am J Pathol. 2000;156:361-81.

38. Brooks SA, Lomax-Browne HJ, Carter TM, Kinch CE, Hall DMS. Molecular interactions in cancer cell metastasis. Acta Histochem. 2010;112:3-25.

39. Tang J, Wang J, Fan L, Li X, Liu N, Luo W, et al. cRGD inhibits vasculogenic mimicry formation by down-regulating uPA expression and reducing EMT in ovarian cancer. Oncotarget. 2016;7:24050-62.

40. Chillà A, Margheri F, Biagioni A, Del Rosso M, Fibbi G, Laurenzana A. Mature and progenitor endothelial cells perform angiogenesis also under protease inhibition: the amoeboid angiogenesis. J Exp Clin Cancer Res. 2018;37:74.

41. Hsiao YH, Su SC, Lin CW, Chao YH, Yang WE, Yang SF. Pathological and therapeutic aspects of matrix metalloproteinases: implications in childhood leukemia. Cancer Metastasis Rev. 2019;38:829-37.

42. Andreasen PA, Egelund R, Petersen HH. The plasminogen activation system in tumor growth, invasion, and metastasis. Cell Mol Life Sci. 2000;57:25-40. 
43. de Vries TJ, Quax PH, Denijn M, Verrijp KN, Verheijen JH, Verspaget HW, et al. Plasminogen activators, their inhibitors, and urokinase receptor emerge in late stages of melanocytic tumor progression. Am J Pathol. 1994;144:70-81.

44. Rofstad EK, Rasmussen H, Galappathi K, Mathiesen B, Nilsen K, Graff BA. Hypoxia promotes lymph node metastasis in human melanoma xenografts by up-regulating the urokinase-type plasminogen activator receptor. Cancer Res. 2002;62:1847-53.

45. Besch R, Berking C, Kammerbauer C, Degitz K. Inhibition of urokinase-type plasminogen activator receptor induces apoptosis in melanoma cells by activation of p53. Cell Death Differ. 2007;14:818-29.

46. Matheis F, Heppt MV, Graf SA, Düwell P, Kammerbauer C, Aigner A, et al. A bifunctional approach of immunostimulation and UPAR inhibition shows potent antitumor activity in melanoma. J Invest Dermatol. 2016;136:2475-84.

47. Mirmohammadsadegh A, Mota R, Gustrau A, Hassan M, Nambiar S, Marini A, et al. ERK1/2 is highly phosphorylated in melanoma metastases and protects melanoma cells from cisplatin-mediated apoptosis. J Invest Dermatol. 2007;127:2207-15.

48. Treps L, Faure S, Clere N. Vasculogenic mimicry, a complex and devious process favoring tumorigenesis-interest in making it a therapeutic target. Pharmacol Ther. 2021;223:107805.

49. Hendrix MJ, Seftor EA, Seftor RE, Chao JT, Chien DS, Chu YW. Tumor cell vascular mimicry: novel targeting opportunity in melanoma. Pharmacol Ther. 2016;159:83-92.

50. D’Aguanno S, Mallone F, Marenco M, Del Bufalo D, Moramarco A. Hypoxia-dependent drivers of melanoma progression. J Exp Clin Cancer Res. 2021;40:159.

51. Seftor EA, Meltzer PS, Schatteman GC, Gruman LM, Hess AR, Kirschmann DA, et al. Expression of multiple molecular phenotypes by aggressive melanoma tumor cells: role in vasculogenic mimicry. Crit Rev Oncol Hematol. 2002;44:17-27.

52. Bonneau B, Prudent J, Popgeorgiev N, Gillet G. Non-apoptotic roles of Bcl-2 family: the calcium connection. Biochim Biophys Acta. 2013;1833:1755-65.

53. Morris JL, Gillet G, Prudent J, Popgeorgiev N. Bcl-2 family of proteins in the control of mitochondrial calcium signalling: an old chap with new roles. Int J Mol Sci. 2021;22:3730.

54. Justino AB, Florentino RM, França A, Filho ACML, Franco RR, Saraiva AL, et al. Alkaloid and acetogenin-rich fraction from Annona crassiflora fruit peel inhibits proliferation and migration of human liver cancer HepG2 cells. PLoS One. 2021;16:e0250394.

55. D’Aguanno S, Del Bufalo D. Inhibition of anti-apoptotic Bcl-2 proteins in preclinical and clinical studies: current overview in cancer. Cells. 2020;9:1287.

56. Merino D, Kelly GL, Lessene G, Wei AH, Roberts AW, Strasser A. BH3-mimetic drugs: blazing the trail for new cancer medicines. Cancer Cell. 2018;34:879-91.

57. Inoue-Yamauchi A, Jeng PS, Kim K, Chen HC, Han S, Ganesan YT, et al. Targeting the differential addiction to anti-apoptotic BCL-2 family for cancer therapy. Nat Commun. 2017;8:16078. 